\title{
Stochastic resonance of fractional-order Langevin equation driven by periodic modulated noise with mass fluctuation
}

Shan Yang ${ }^{1}$, Mou Deng ${ }^{2}$ and Ruibin Ren ${ }^{3 *}$

Correspondence:

Airy_Ren@163.com

${ }^{3}$ College of Mathematics,

Southwest Jiaotong University,

Chdengdu, China

Full list of author information is

available at the end of the article

\section{를 Springer}

\begin{abstract}
The stochastic resonance (SR) of a second-order harmonic oscillator subject to mass fluctuation and periodic modulated noise in viscous media is studied. The mass fluctuation noise is modeled as dichotomous noise and the memory of viscous media is characterized by fractional power kernel function. By using the Shapiro-Loginov formula and Laplace transform, we got the analytical expression of the first moment of the steady-state response and studied the relationship between the system response and the system parameters in the long-time limit. The simulation results show the non-monotonic dependence between the response amplitude and the input signal frequency, noise parameters of the system, etc, which indicates that the bona fide resonance and the generalized SR phenomena appear. Furthermore, the mass fluctuation noise, modulation noise, and the fractional order work together, producing more complex dynamic phenomena than the integral-order system. For example, there is a transition from bimodal resonance to unimodal resonance between the amplitude and the driving frequency under different fractional orders.
\end{abstract}

Keywords: Fractional Langevin equation; Stochastic resonance; Mass fluctuation; Signal modulated noise

\section{Introduction}

As the research frontier of the statistical physics and the stochastic dynamical system, the stochastic resonance (SR) driven by fluctuation and periodic signal recently become a popular research direction [1-4]. The term of SR was proposed by Benzi [5, 6] and Nicolis [7] to explain the climatic mechanics of periodic glaciers in the 1980s. Contrary to the common knowledge that noise is harmful, the SR phenomenon shows that random disturbance (noise) can produce a cooperative effect under certain conditions, it can realize the transfer of noise energy to signal energy, and it thus may strengthen the system output. Since then, more and more scholars have paid attention to the theoretical and experimental researches on SR, which makes it gradually become a hot topic in the field of stochastic dynamics.

To avoid ambiguity, the SR mentioned in this paper is the generalized SR without a special explanation. That is, the non-monotonic transformation phenomenon of some func-

(c) The Author(s) 2020. This article is licensed under a Creative Commons Attribution 4.0 International License, which permits use, sharing, adaptation, distribution and reproduction in any medium or format, as long as you give appropriate credit to the original author(s) and the source, provide a link to the Creative Commons licence, and indicate if changes were made. The images or other third party material in this article are included in the article's Creative Commons licence, unless indicated otherwise in a credit line to the material. If material is not included in the article's Creative Commons licence and your intended use is not permitted by statutory regulation or exceeds the permitted use, you will need to obtain permission directly from the copyright holder. To view a copy of this licence, visit http://creativecommons.org/licenses/by/4.0/ 
tions of system response (such as moment, power spectrum, autocorrelation function, signal to noise ratio, etc.) with some characteristic parameters of the system (such as frequency, excitation amplitude or noise intensity, correlation rate) [8].

In the past 30 years, a large part of the research on the SR phenomena has been carried out around different dynamical systems and noise forms, and corresponding physical models have been established, respectively. From the perspective of the model, fluctuations enter the model equation in the form of multiplication, so the study of the SR phenomenon of the harmonic oscillator system essentially belongs to the study of the dynamic behavior of the resonant subsystem under multiplicative external noise [9-11]. However, we found that in most previous work, it is generally considered that the external noise of harmonic oscillator caused by the disturbance of system damping or natural frequency $[3,11,12]$, while the external noise caused by the perturbation of the oscillator mass is seldom mentioned.

In recent years, the SR phenomenon of harmonic oscillator systems with mass fluctuation has attracted considerable attention from many scholars. Here, the so-called mass fluctuation refers to that in many chemical and biological systems, some molecules in the medium have certain adsorption capacity and will randomly absorb and desorb on Brownian particles, so that the mass of the system is no longer a constant, but a random value. A growing number of studies show that in some practical biological, physical and engineering fields, studying the dynamic behaviors of a system with mass fluctuation can better depict the real physical scene, and thus obtain a possibly richer physical explanation. Łuczka [13] studied stochastic growth quality cluster diffusion phenomenon and points out that the cluster center of mass of the azimuth shift of anomalous behavior is a function of cluster size. Gitterman [14] considered the random resonance phenomenon of a simple harmonic oscillator with random mass, and the model actually corresponds to a new type of Brownian motion.

At the same time, some scholars start to consider the SR phenomenon of a linear system driven by the periodic signal modulation noise which is common in physical engineering systems [15]. In the process of signal measurement, the stronger the intensity of the measured signal, the stronger the measurement noise is accompanied by it. Therefore, the measurement noise and the signal are not completely independent. In many practical systems, such as extenders in optics and radio astronomy, input signals and external noise act on the system multiplicatively, that is, the noise is modulated by periodic signals [16]. Therefore, one of the main works of this paper will be to consider the SR phenomenon of harmonic oscillator with mass fluctuation under the effect of periodic modulation noise. Such a model has not been reported in the existing literature.

On the other hand, as a natural generalization of integral-order stochastic dynamical system, the research of SR based on fractional-order stochastic dynamical system gradually attracted great interest [17-19]. In fact, the friction term in the classical Langevin equation is only dependent on the current speed, which is modeled as $-\gamma x(t)$. For most of the real systems, such as viscoelastic processes, however, the damping term no longer depends only on the current speed but also the historical speed, i.e., one has to take into account the memory effects. Meanwhile, the fractional derivative, naturally has a good ability to describe such memory property [20-27]. Therefore, we proposed a power-law kernel function to model such processes which are associated with the Caputo fractional derivative. 
Accordingly, the classical Langevin equation are generalized to the fractional Langevin equation (FLE) $[28,29]$. Typical examples of such systems include viscoelastic media, disordered semiconductors, dense polymer solutions, and colloidal glasses. In recent years, the research of the SR phenomenon in FLE has drawn great attention due to the intensive development of the theory of fractional calculus and the application of FLE in modeling many physical phenomena. The literature indicates that the FLE has much richer dynamic behaviors than the classical harmonic oscillator and is more beneficial to the description of real environments. Some scholars have also made attempts and explorations in this respect, and studies have shown that fractional dynamical systems based on fractional-order differential equations tend to have more abundant dynamic phenomena than integerorder stochastic dynamical systems under similar conditions [30-34].

The structure of the paper is as follows. In Sects. 2, 3 and 4, we introduce the model of the fractional Langevin equations and then determine the related explicit results. In Sect. 5, we analyze the collective behavior of the fractional Langevin equations with the explicit results. In Sect. 6, some discussions conclude this paper.

\section{Fractional Langevin equation}

\subsection{Generalized Langevin equation}

We address the second-order Langevin equation in a classic thermal fluctuation environment:

$$
m \ddot{x}+\gamma \dot{x}=F(x, t)+\eta(t)
$$

where $F(x, t)$ is the external driving force, $\eta(t)$ is the internal noise, $\gamma \dot{x}$ is the damping force. The model depicts the motion of Brown particles in an ideal liquid, however, in an inhomogeneous solution, the damping force on the particles will change with time, which is usually expressed by the following generalized Langevin equation:

$$
m \ddot{x}+\gamma \int_{0}^{t} \beta(t-s) \dot{x}(s) \mathrm{d} s=F(x, t)+\eta(t),
$$

here, $\gamma$ is the damping coefficient, $\beta(t)$ is the damping kernel function, which is used to characterize the time-dependent damping changes of heterogeneous media.

\subsection{Fractional Langevin equation}

In many physical, biological and chemical systems, the movement of Brown particles in viscous media is often a rather complex problem. In such viscous media, the damping force of particles often shows the memory of historical velocity, Mankin and Soika, etc. modeled the damping kernel function by a power law as follows:

$$
\beta(t)=\frac{|t|^{-\alpha}}{\Gamma(1-\alpha)} .
$$

Substituting the above equation into the generalized Langevin equation (2), we get the fractional Langevin equation:

$$
m \ddot{x}+\gamma \int_{0}^{t} \frac{|t-s|^{-\alpha}}{\Gamma(1-\alpha)} \dot{x}(s) \mathrm{d} s=m \ddot{x}+D^{\alpha} x=F(x, t)+\eta^{\alpha}(t),
$$


here $D^{\alpha}$ represent the Caputo fractional calculus operator [20], $\alpha$ is the fractional order. $\eta^{\alpha}(t)$ is the fractional noise, which satisfied the fluctuation-dissipation theorem with damping kernel function $\beta(t)$.

\section{System model and its solution}

A fractional harmonic oscillator model with mass fluctuation driven by periodic modulation noise, can be described by the fractional Langevin equation as follows:

$$
(m+\xi(t)) \frac{\mathrm{d}^{2} x(t)}{\mathrm{d} t^{2}}+\gamma D^{\alpha} x(t)=-m \omega^{2} x(t)+A_{0} \cos (\Omega t) \xi(t)+\eta^{\alpha}(t),
$$

where $m$ is the mass of the particle, $\gamma$ is the damping coefficient, $\omega$ is the inherent frequency, $A_{0}, \Omega$ are the amplitude and the frequency of the external driving signal, respectively, $\eta^{\alpha}(t)$ is the additive internal noise related with the system order $\alpha, \xi(t)$ is the multiplicative external noise, here we build it in a dichotomous way, $\xi(t)=\sigma_{1}$ or $-\sigma_{1},\left(\sigma_{1}>0\right)$, the external noise satisfied the following properties:

$$
\begin{aligned}
& \langle\xi(t)\rangle=0, \\
& \langle\xi(t) \xi(s)\rangle=\langle\xi(s) \xi(t)\rangle=\sigma_{1}{ }^{2} \exp \left[-\lambda_{1}|t-s|\right] .
\end{aligned}
$$

Since the mass cannot be negative, which means $\left(m \pm \sigma_{1}\right)>0$, we let the mass fluctuation noise intensity always satisfy the condition:

$$
\sigma_{1}<m
$$

$\eta^{\alpha}(t)$ is the fractional Gaussian noise, which is the generalized derivative of the fractional Brown motion $B^{\alpha}(t)$, and they satisfy

$$
\left\langle\eta^{\alpha}(t)\right\rangle=0
$$

$\eta^{\alpha}(t)$ is the random fluctuation caused by thermal motion of environmental molecules, its autocorrelation function satisfies Kubo's second fluctuation-dissipation theorem [35]:

$$
\left\langle\eta^{\alpha}(t) \eta^{\alpha}(s)\right\rangle=k_{B} T \gamma(t-s)=k_{B} T \gamma \frac{|t-s|^{-\alpha}}{\Gamma(1-\alpha)} .
$$

In this paper, we assume that dependence between the external noise and internal noise due to its different origins:

$$
\left\langle\xi(t) \eta^{\alpha}(s)\right\rangle=\left\langle\eta^{\alpha}(t) \xi(s)\right\rangle=0
$$

\section{The first moment and response amplitude}

\subsection{Fractional Shapiro-Loginov formula}

In many practical physics and engineering system models, noise is one of the inevitable factors to be considered. When the noise is modeled as a steady-state stochastic process, how to deal with the correlation between random variables is the key to solve the stochastic differential equation (SDE) of the system. Shapiro-Loginov formula is an effective tool for solving such SDEs with exponential correlation random terms: 
Theorem 4.1 ([36], Shapiro-Loginov formula) If a stationary random process $\xi(t)$ satisfies the following statistical properties:

$$
\langle\xi(t)\rangle=0 ; \quad\left\langle\xi\left(t_{1}\right) \xi\left(t_{2}\right)\right\rangle=\sigma^{2} \exp \left[-v\left|t_{1}-t_{2}\right|\right] .
$$

Meanwhile if $\phi(t)$ is the function of $\xi(t)$, then we have

$$
\frac{\mathrm{d}}{\mathrm{d} t}\langle\xi(t) \phi(t)\rangle=\left\langle\xi(t) \frac{\mathrm{d}}{\mathrm{d} t} \phi(t)\right\rangle-v\langle\xi(t) \phi(t)\rangle .
$$

The Shapiro-Loginov formula gives a very simple method to deal with the correlation between random variables. The unity of equation variables can be realized by using the Shapiro-Loginov formula, so as to solve the equation.

In the fractional-order stochastic differential equation (FSDE), the classical ShapiroLoginov formula needs to be generalized:

Theorem 4.2 ([32], Fractional Shapiro-Loginov formula) Assume that the following assumptions hold:

1. $\langle\xi(t)\rangle=0,\left\langle\xi\left(t_{1}\right) \xi\left(t_{2}\right)\right\rangle=\sigma^{2} \exp \left[-v\left|t_{1}-t_{2}\right|\right]$.

2. $\phi(t)$ is a function of $\xi(t)$.

Then we have the following fractional Shapiro-Loginov formula:

$$
\left\langle\xi(t) D^{\alpha} \phi(t)\right\rangle=e^{-\nu t} D^{\alpha}\left(|\xi(t) \phi(t)\rangle e^{\nu t}\right) .
$$

Here $D^{\alpha}$ is the Caputo fractional calculus operator, $0<\alpha \leq 1$.

It is easy to verify that when the fractional order $\alpha=1$, the fractional Shapiro-Loginov formula degenerates into the classical integer-order Shapiro-Loginov formula.

\subsection{Steady-state response of the first moment and output amplitude}

Averaging the fractional Langevin equation (5) yields

$$
m \frac{\mathrm{d}^{2}\langle x(t)\rangle}{\mathrm{d} t^{2}}+\left\langle\xi(t) \frac{\mathrm{d}^{2}\langle x(t)\rangle}{\mathrm{d} t^{2}}\right\rangle+\gamma D^{\alpha}\langle x(t)\rangle=-m \omega^{2}\langle x(t)\rangle .
$$

Combining with the Shapiro-Loginov formula,

$$
\left\langle\xi(t) \frac{\mathrm{d}^{n} x}{\mathrm{~d} t^{n}}\right\rangle=\left(\frac{\mathrm{d}}{\mathrm{d} t}+\lambda_{1}\right)^{n}\langle\xi(t) x\rangle
$$

Substituting (16) into (15) yields

$$
m \frac{\mathrm{d}^{2}\langle x(t)\rangle}{\mathrm{d} t^{2}}+\left(\frac{\mathrm{d}}{\mathrm{d} t}+\lambda_{1}\right)^{2}\langle\xi(t) x(t)\rangle+\gamma D^{\alpha}\langle x(t)\rangle=-m \omega^{2}\langle x(t)\rangle .
$$

Multiplying (5) by $\xi(t)$, and averaging the obtained equation, we have:

$$
\begin{aligned}
& \sigma_{1}^{2} \frac{\mathrm{d}^{2}\langle x(t)\rangle}{\mathrm{d} t^{2}}+m\left(\frac{\mathrm{d}}{\mathrm{d} t}+\lambda_{1}\right)^{2}\langle\xi(t) x(t)\rangle+\gamma\left\langle\xi(t) D^{\alpha} x(t)\right\rangle \\
& \quad=-m \omega^{2}\langle\xi(t) x(t)\rangle+A_{0} \cos (\Omega t) \sigma_{1}^{2} .
\end{aligned}
$$


Considering the fractional Shapiro-Loginov formula:

$$
\left\langle\xi(t) D^{\alpha} x(t)\right\rangle=\mathrm{e}^{-\lambda_{1} t} D^{\alpha}\left(\left|\xi(t) x(t) \mathrm{e}^{\lambda_{1} t}\right\rangle\right) .
$$

Substituting (19) into (18) yields

$$
\begin{aligned}
& \sigma_{1}^{2} \frac{\mathrm{d}^{2}\langle x(t)\rangle}{\mathrm{d} t^{2}}+m\left(\frac{\mathrm{d}}{\mathrm{d} t}+\lambda_{1}\right)^{2}\langle\xi(t) x(t)\rangle+\gamma \mathrm{e}^{-\lambda_{1} t} D^{\alpha}\left(\left|\xi(t) x(t) \mathrm{e}^{\lambda_{1} t}\right\rangle\right), \\
& \quad=-m \omega^{2}\langle\xi(t) x(t)\rangle+A_{0} \cos (\Omega t) \sigma_{1}^{2} .
\end{aligned}
$$

Let $\langle x(t)\rangle=x_{1},\langle\xi(t) x(t)\rangle=x_{2}$, close equations with two variables are found:

$$
\left\{\begin{array}{l}
\left(m \frac{\mathrm{d}^{2}}{\mathrm{~d} t^{2}}+\gamma D^{\alpha}+m \omega^{2}\right) x_{1}(t)+\left(\frac{\mathrm{d}}{\mathrm{d} t}+\lambda_{1}\right)^{2} x_{2}(t)=0 \\
\sigma_{1}^{2} \frac{\mathrm{d}^{2}}{\mathrm{~d} t^{2}} x_{1}(t)+\left(m\left(\frac{\mathrm{d}}{\mathrm{d} t}+\lambda_{1}\right)^{2}+m \omega^{2}\right) x_{2}(t)+\gamma \mathrm{e}^{-\lambda_{1} t} D^{\alpha}\left(\left\langle\xi(t) x(t) \mathrm{e}^{\lambda_{1} t}\right\rangle\right) \\
\quad=A_{0} \cos (\Omega t) \sigma_{1}^{2}
\end{array}\right.
$$

By using the Laplace transform on (21), we have

$$
\left\{\begin{array}{l}
d_{11} X_{1}(s)+d_{12} X_{2}(s)=d_{13} \\
d_{21} X_{1}(s)+d_{22} X_{2}(s)=A_{0} \frac{s}{s^{2}+\Omega^{2}}+d_{23}
\end{array}\right.
$$

where

$$
\left\{\begin{array}{l}
d_{11}=m s^{2}+\gamma s^{\alpha}+m \omega^{2}, \\
d_{12}=\left(s+\lambda_{1}\right)^{2}, \\
d_{13}=\left(m s+\gamma s^{\alpha-1}\right) x_{1}(0)+m \dot{x}_{1}(0)+\left(s+2 \lambda_{1}\right) x_{2}(0)+\dot{x}_{2}(0), \\
d_{21}=\sigma_{1}^{2} s^{2}, \\
d_{22}=m\left(s+\lambda_{1}\right)^{2}+\gamma\left(s+\lambda_{1}\right)^{\alpha}+m \omega^{2}, \\
d_{23}=\sigma_{1}^{2} s x_{1}(0)+\sigma_{1}^{2} \dot{x}_{1}(0)+\left(m s+2 m \lambda_{1}+\gamma\left(s+\lambda_{1}\right)^{\alpha-1}\right) x_{2}(0)+m \dot{x}_{2}(0) .
\end{array}\right.
$$

Combining (22)and (23), we get

$$
\left\{\begin{array}{l}
X_{1}(s)=-\frac{d_{12}}{d_{11} d_{22}-d_{12} d_{21}} A_{0} \frac{s}{s^{2}+\Omega^{2}}+\frac{d_{13} d_{22}-d_{12} d_{23}}{d_{11} d_{22}-d_{12} 2 d_{21}}, \\
X_{2}(s)=\frac{d_{11}}{d_{11} d_{22}-d_{12} d_{21}} A_{0} \frac{s}{s^{2}+\Omega^{2}}+\frac{d_{11} d_{23}-d_{21} d_{13}}{d_{11} d_{22}-d_{12} d_{21}}
\end{array}\right.
$$

On the other hand, from the aspect of signal processing, the system response can be regarded as the output of the linear time-invariant system in which the input signal $A_{0} \cos (\Omega t)$ passes through the transfer function $H_{10}$, here

$$
H_{10}=-\frac{d_{12}}{d_{11} d_{22}-d_{12} d_{21}} .
$$

Here we consider the long-time limit situation, then the effect of the initial conditions is almost negligible,

$$
\langle x(t)\rangle_{a s}=\left\langle\left. x(t)\right|_{t \rightarrow \infty} \equiv A_{0} \int_{0}^{t} h_{i 0}\left(t-t^{\prime}\right) \cos \left(\Omega t^{\prime}\right) \mathrm{d} t^{\prime} .\right.
$$


According to the properties of the linear time-invariant system, the steady-state response of the system has the following form:

$$
\langle x(t)\rangle_{a s}=A \cos (\Omega t+\phi) .
$$

From the system transfer function $H_{10}$, the expressions of the steady-state response amplitude and phase shift of the system can be obtained:

$$
\left\{\begin{array}{l}
A=A_{0}\left|H_{10}(j \Omega)\right|=A_{0} \sqrt{\frac{f_{1}{ }^{2}+f_{2}{ }^{2}}{f_{3}{ }^{2}+f_{4}}}, \\
\phi=\arg \left(H_{10}(j \Omega)\right)=\arctan \left(\frac{f_{2} f_{3}-f_{1} f_{4}}{f_{1} f_{3}+f_{2} f_{4}}\right),
\end{array}\right.
$$

where

$$
\begin{aligned}
f_{1}= & \Omega^{2}-\lambda_{1}^{2}, \\
f_{2}= & -2 \lambda_{1} \Omega, \\
p_{1}= & m \omega^{2}-m \Omega^{2}+\gamma \Omega^{\alpha} \cos (\alpha \pi / 2), \\
p_{2}= & m \omega^{2}+\gamma r^{\alpha} \cos (\alpha \theta), \\
f_{3}= & -\left(m p_{1}+\sigma_{1}^{2} \Omega^{2}\right) f_{1}+m \gamma \Omega^{\alpha} \sin (\alpha \pi / 2) f_{2} \\
& +p_{1} p_{2}-\gamma^{2}(r \Omega)^{\alpha} \sin (\alpha \pi / 2) \sin (\alpha \theta), \\
f_{4}= & -m \gamma \Omega^{\alpha} \sin (\alpha \pi / 2) f_{1}+-\left(m p_{1}+\sigma_{1}^{2} \Omega^{2}\right) f_{2} \\
& +\gamma r^{\alpha} \sin (\alpha \theta) p_{1}+p_{2} \gamma \Omega^{\alpha} \sin (\alpha \pi / 2), \\
r= & \sqrt{\Omega^{2}+\lambda_{1}^{2},} \quad \theta=\arg \tan \left(\Omega / \lambda_{1}\right) .
\end{aligned}
$$

\section{Simulation results and analysis}

In the last section, the analytical expression of the steady-state response of the system was obtained. Next, we will discuss the stochastic resonance phenomena between the steadystate response amplitude and random noise intensity $\sigma_{1}$, noise correlation rate $\lambda$, fractional order $\alpha$, damping coefficient $\gamma$ and oscillator mass $m$ in combination with the simulation results.

\subsection{The effect of multiplicative external noise on the bona fide SR}

Figures 1 and 2 respectively depict the curves of system steady-state response amplitude $A$ as a function of external driven frequency $\Omega$ with different noise intensity $\sigma_{1}$ and correlation rate $\lambda$. As the frequency of the periodic input signal increases, $A$ appears as a non-monotonic variation, which indicates the bona fide SR appears in the system. As the noise intensity increases, the amplitude curve changes from unimodal resonance to bimodal resonance, and the maximum value gradually decreases, and the resonance peak gradually shifts to the right. In Fig. 2, with the increase of noise correlation rate $\sigma_{1}$, the amplitude curve changes from bimodal resonance to unimodal resonance. This is because the correlation rate of two-state noise is the inverse of the conversion rate, the smaller the correlation rate, the higher the conversion rate, thus causing the system to exhibit a novel double-peak resonance phenomenon. 
Figure 1 A as a function of $\Omega, m=1, \gamma=0.2, \omega=1$, $\alpha=0.6, \lambda=0.4$

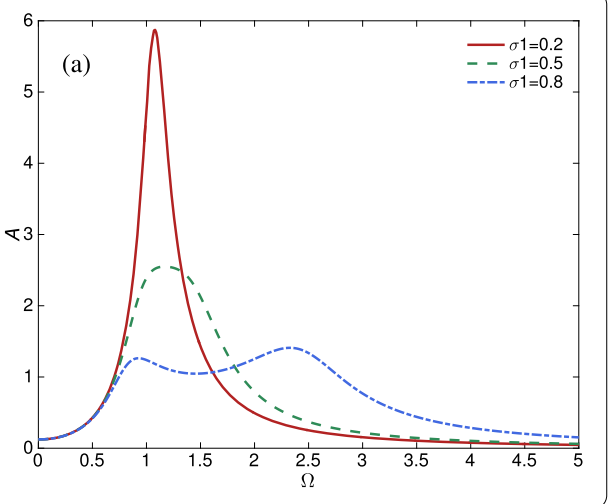

Figure $2 A$ as a function of $\Omega, m=1, \gamma=0.2, \omega=1$, $\alpha=0.6, \lambda=0.4, \sigma_{1}=0.6$

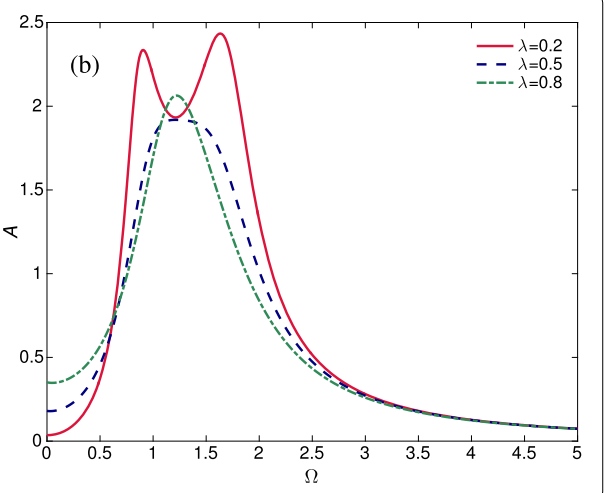

The above results show that in the case of a fractional-order system, the synergistic effect of dichotomous noise and periodic modulation noise makes the noise energy transfer to signal energy, thus enhancing the output of the system, making the system show rich real resonance phenomena such as single-peak resonance and double-peak resonance.

\subsection{The influence of damping characteristics on the bona fide SR}

Figures 3 and 4, respectively, depict the curves of system steady-state response amplitude $A$ as a function of external driving frequency $\Omega$ with different fractional order $\alpha$ and damping coefficient $\gamma$ When $\alpha=0.1$, the steady-state response amplitude $A$ shows a bimodal SR behavior, and the second resonance-peak value is larger than the first one, with the increase of $\alpha$, the bimodal SR gradually turns into unimodal SR, and the maximum value decreases, and the peak position gradually shifts to the left. In Fig. 4, with the increase of $\gamma$, the resonance peak of the amplitude curve gradually flattens out, and the resonance maximum value gradually decreases. This shows that the increase of the damping coefficient weakens the SR phenomenon of the steady-state response of the system.

The above results show that the damping characteristics of the harmonic oscillator can cause the system steady-state response amplitude produced abundant bona fide SR under certain conditions, and the form of this SR is closely related to the damping coefficient. In particular, when the fractional-order system and damping coefficient are small, the system will show the double-peak SR phenomenon which the traditional harmonic oscillator model does not have. 
Figure $3 A$ as a function of $\Omega$, The other parameters are $m=1, \omega=1, \sigma=0.6, \lambda=0.4, \gamma=0.6$

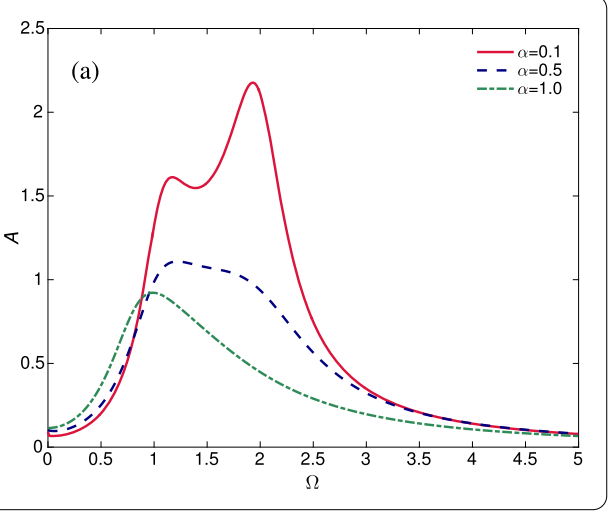

Figure 4 A as a function of $\Omega$, The parameters are $m=1, \omega=1, \sigma=0.6, \lambda=0.4, \alpha=0.6$

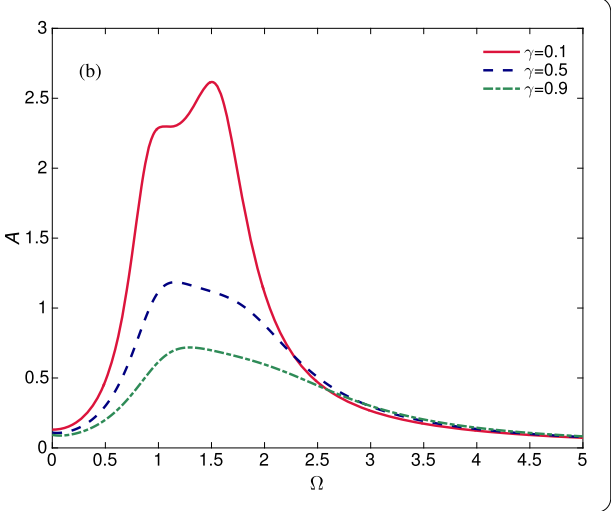

Figure $5 A$ as a function of $\sigma_{1}$, The parameters are $m=1, \gamma=0.2, \omega=1, \Omega=1.6, \lambda=0.4$

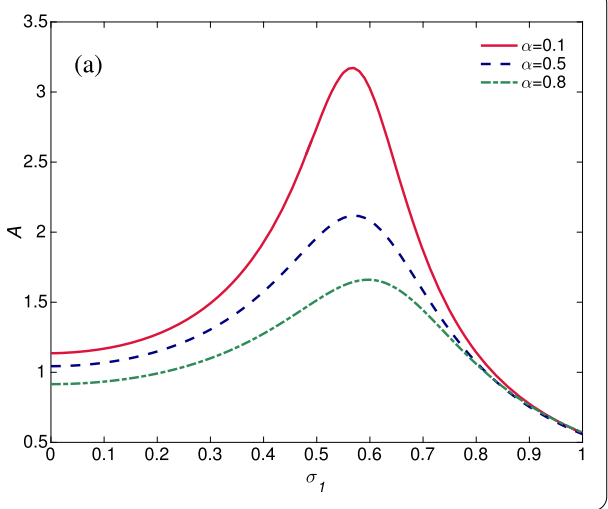

\subsection{The influence of other parameters on SR}

Figures 5 and 6, respectively, mapped the system steady-state response amplitude $A$ as a function of the noise strength $\sigma_{1}$. From the curves of different fractional order $\alpha$ and noise correlation rate $\lambda$, it can be seen that in both cases, $A$ shows a non-monotonic dependence on noise intensity, that is, the SR phenomenon appears. With the increase of $\alpha$ and $\lambda$, the SR maximum value decreases and the formant position gradually shifts to the right.

The above results show that the fractional-order number and the noise correlation rate are significant to the phenomenon of stochastic resonance of the system. Different $\alpha$ and $\lambda$ will increase or decrease the maximum value of the resonance peak, and the peak po- 
Figure 6 as a function of $\sigma_{1}$, The parameters are $m=1, \gamma=0.2, \omega=1, \Omega=1.6, \alpha=0.6$

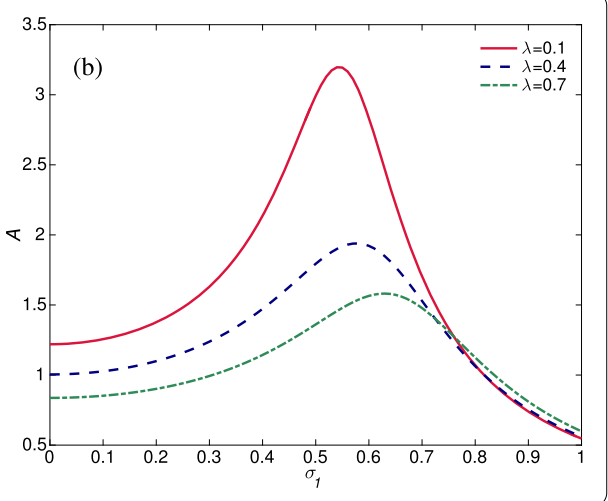

Figure $7 A$ as a function of $m$, The parameters are $\gamma=0.2, \omega=1, \sigma=0.6, \Omega=1.6, \lambda=0.4$

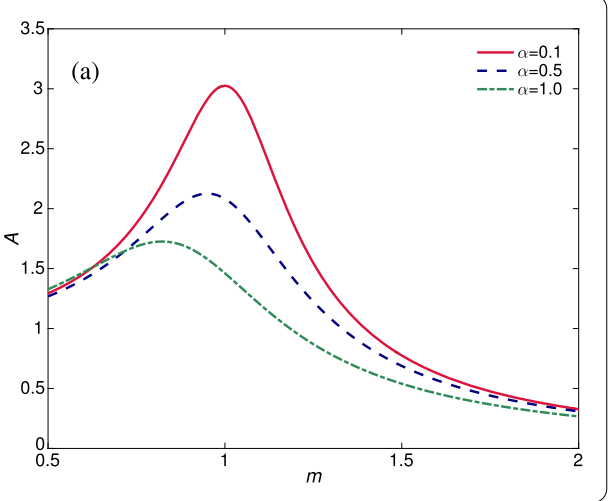

sition will appear earlier or later. Therefore, the SR phenomenon of such systems can be effectively controlled by adjusting the memorization and the correlation rate of noise.

Finally, we draw the curve of system steady-state response amplitude $A$ as a function of oscillator mass $m$. From the curves of different fractional order $\alpha$ and noise correlation rate $\lambda$, it can be seen that in Fig. 7, $A$ appears parametric induced resonance with the change of $m$. With the increase of fractional order, the resonant-peak value decreases and the position of the resonant peak moves to the left gradually. This indicates that the memory characteristics of the system play an important role in the SR phenomenon: the smaller the $\alpha$ is, the stronger the system memory is, and the longer the contribution of history to the present moment is, thus enhancing the SR phenomenon of the system's steady-state response. In Fig. 8, with the increase of $\lambda$, the resonance-peak value first decreases and then increases, and the position of the resonance peak gradually shifts to the left.

The above results show that the steady-state response amplitude $A$ and the mass of the oscillator $m$ has obvious parametric induced SR phenomenon. In addition, the memorization of the system can promote the occurrence of SR of the system to a certain extent, while the increase of noise correlation rate $\lambda$ first inhibits and then enhances the stochastic resonance of the system.

\section{Conclusion}

The mass fluctuation noise and periodic modulation noise exist widely in various complex physical and engineering systems. In this paper, the fractional-order mass fluctuation harmonic oscillator model driven by periodic modulation noise is proposed and established 
Figure $8 A$ as a function of $m$, The parameters are $\gamma=0.2, \omega=1, \sigma=0.6, \Omega=1.6, \alpha=0.6$

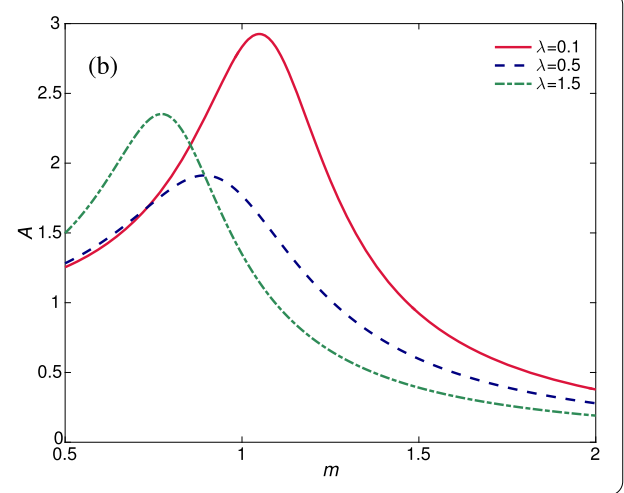

for the first time. On this basis, the influences of external noise parameters, system damping parameters and other model parameters on SR of the system are deeply studied. We found that due to external noise, memory characteristics and system synergy, the stable response of the system appears as a diversified SR phenomenon. By adjusting some parameters of the model, we can effectively control the SR phenomenon of the second-order linear systems within a certain range, which provides another new perspective for the application of stochastic resonance theory in practical physics and engineering.

\section{Funding}

This work was supported by the Fundamental Research Funds for the Central Universities, China (Grant No. 2682018CX65).

\section{Competing interests}

The authors declare that they have no competing interests.

\section{Authors' contributions}

All authors contributed equally to this work. All authors read and approved the final manuscript.

\section{Author details}

${ }^{1}$ Department of Computer \& Software, Jincheng College of Sichuan University, Chengdu, China. ${ }^{2}$ Sichuan Institute of Industrial Technology, Deyang, China. ${ }^{3}$ College of Mathematics, Southwest Jiaotong University, Chdengdu, China.

\section{Publisher's Note}

Springer Nature remains neutral with regard to jurisdictional claims in published maps and institutional affiliations.

Received: 24 July 2019 Accepted: 2 January 2020 Published online: 21 February 2020

\section{References}

1. Berdichevsky, V., Gitterman, M.: Stochastic resonance in linear systems subject to multiplicative and additive noise. Phys. Rev. E 60(2), 1494-1499 (1999)

2. Kosińska, I.: Stochastic resonance in discrete kinetics with delay. Physica A 325(1-2), 116-123 (2003)

3. Guo, F., Li, H., Liu, J., et al.: Stochastic resonance in a linear system with random damping parameter driven by trichotomous noise. Physica A 409(3), 1-7 (2014)

4. Li, J.H., Han, Y.X.: Phenomenon of stochastic resonance caused by multiplicative asymmetric dichotomous noise. Phys. Rev. E, Stat. Nonlinear Soft Matter Phys. 74(5 Pt. 1), 121 (2006)

5. Benzi, R., Sutera, A., Vulpiani, A.: The mechanism of stochastic resonance. J. Phys. A 14(11), L453-L457 (1981)

6. Benzi, R., Parisi, G., Sutera, A., et al.: Stochastic resonance in climatic change. Tellus 34(1), 10-16 (1982)

7. Nicolis, C.: Stochastic aspects of climatic transitions - response to a periodic forcing. Tellus 34(2), 108-110 (1982)

8. Gitterman, M.: Classical harmonic oscillator with multiplicative noise. Physica A 352(s2-4), 309-334 (2005)

9. Gitterman, M., Klyatskin, V.I.: Brownian motion with adhesion: harmonic oscillator with fluctuating mass. Phys. Rev. E 81(1), 561 (2010)

10. Gitterman, M.: Mean-square displacement of a stochastic oscillator: linear vs quadratic noise. Physica A 391(11), 3033-3042 (2012)

11. Gitterman, M.: Oscillator with random trichotomous mass. Physica A 391(22), 5343-5348 (2012)

12. He, G., Tian, Y., Luo, M.: Stochastic resonance in an underdamped fractional oscillator with signal-modulated noise. J. Stat. Mech. 2014, P05018 (2014)

13. Luczka, J., Hanggi, P., Gadomski, A.: Diffusion of clusters with randomly growing masses. Phys. Rev. E 51(6), 5762-5769 (1995)

14. Gitterman, M.: Stochastic oscillator with random mass: new type of Brownian motion. Physica A 395(4), 11-21 (2014) 
15. Portman, J., Khasin, M., Shaw, S.W., et al.: The spectrum of an oscillator with fluctuating mass and nanomechanical mass sensing. In: APS March Meeting. American Physical Society (2010)

16. Cao, L., Wu, D.J.: Stochastic resonance in a linear system with signal-modulated noise. Europhys. Lett. 61(5), 593-598 (2007)

17. Lin, L.F., Chen, C., Zhong, S.C., et al.: Stochastic resonance in a fractional oscillator with random mass and random frequency. J. Stat. Phys. 160(2), 497-511 (2015)

18. Soika, E., Mankin, R., Ainsaar, A.: Resonant behavior of a fractional oscillator with fluctuating frequency. Phys. Rev. E 81(1), $132(2012)$

19. Yu, T., Zhang, L., Luo, M.K.: Stochastic resonance in the fractional Langevin equation driven by multiplicative noise and periodically modulated noise. Phys. Scr. 88(4), 045008 (2013)

20. Podlubny, l.: Fractional Differential Equations. Academic Press, San Diego (1999)

21. Shiri, B., Baleanu, D.: System of fractional differential algebraic equations with applications. Chaos Solitons Fractals 120, 203-212 (2019)

22. Baleanu, D., Shiri, B., Srivastave, H.M., Al Qurashi, M.: A Chebyshev spectral method based on operational matrix for fractional differential equations involving non-singular Mittag-Leffler kernel. Adv. Differ. Equ. 2018, 353 (2018)

23. Baleanu, D., Shiri, B.: Collocation methods for fractional differential equations involving non-singular kernel. Chaos Solitons Fractals 116, 136-145 (2018)

24. Agarwal, R.P., Baleanu, D., Hedayati, V., Rezapour, S.: Two fractional derivative inclusion problems via integral boundary condition. Appl. Math. Comput. 257, 205-212 (2015)

25. Baleanu, D., Mousalou, A., Rezapour, S.: On the existence of solutions for some infinite coefficient-symmetric Caputo-Fabrizio fractional integro-differential equations. Bound. Value Probl. 2017(1), 145 (2017)

26. Kojabad, E., Rezapour, S.: Approximate solutions of a sum-type fractional integro-differential equation by using Chebyshev and Legendre polynomials. Adv. Differ. Equ. 2017, 351 (2017)

27. Aydogam, M.S., Baleanu, D., Mousalou, A., Rezapour, S.: On high order fractional integro-differential equations including the Caputo-Fabrizio derivative. Bound. Value Probl. 2018, 90 (2018)

28. Veeresha, P., Prakasha, D.G., Baleanu, D.: An efficient numerical technique for the nonlinear fractional Kolmogorov-Petrovskii-Piskunov equation. Mathematics 7(3), 265 (2019)

29. Baleanu, D., Fernandez, A.: On fractional operators and their classifications. Mathematics 7(9), 830 (2019)

30. Wu, G.C., Deng, Z.G., Baleanu, D., Zeng, D.Q.: New variable-order fractional chaotic systems for fast image encryption. Chaos 29, 083103 (2019)

31. Wu, G.C., Deng, Z.G., Baleanu, D.: Fractional impulsive differential equations: exact solutions, integral equations and short memory case. Fract. Calc. Appl. Anal. 22, 180-192 (2019)

32. Zhong, S., Ma, H., Peng, H., et al.: Stochastic resonance in a harmonic oscillator with fractional-order external and intrinsic dampings. Nonlinear Dyn. 82(1-2), 1-11 (2015)

33. Mourad, K., Fateh, E., Baleanu, D.: Stochastic fractional perturbed control systems with fractional Brownian motion and Sobolev stochastic non local conditions. Collect. Math. 69, 283-296 (2018)

34. Annamalai, A., Kandasamy, B., Baleanu, D., Arumugam, V.: On neutral impulsive stochastic differential equations with Poisson jumps. Adv. Differ. Equ. 2018, 290 (2018)

35. Kubo, R.: The fluctuation-dissipation theorem. Rep. Prog. Phys. 29(1), 255-284 (1966)

36. Shapiro, V.E., Loginov, V.M.: "Formulae of differentiation" and their use for solving stochastic equations. Physica A 91, 563-574 (1978)

\section{Submit your manuscript to a SpringerOpen ${ }^{\circ}$ journal and benefit from:}

- Convenient online submission

- Rigorous peer review

- Open access: articles freely available online

- High visibility within the field

- Retaining the copyright to your article

Submit your next manuscript at $\gg$ springeropen.com 